\title{
Somesthetic spatial generalization in individual rats
}

\author{
SEYMOUR AXELROD ${ }^{2}$ AND PAUL F. KANKOLENSKI
}

STATE UNIVERSITY OF NEW YORK AT BUFFALO

Rats were reinforced for bar pressing in the presence of stimulation of a body locus, and not reinforced for pressing in the absence of stimulation. The response generalized decrementally to stimulation of other loci along the trunk on the side ipsilateral to the training locus. Precise bilateral symmetry of generalization gradients, reported for classically-conditioned dogs, was not found.

Koupalov (1916) and Anrep (1923) reported from Pavlov's laboratory that after a dog had been conditioned to salivate in response to a pricking or vibro-tactile stimulus presented to a locus, 0 , on its body surface, test stimulations of other loci on the same side of the body elicited salivation in amounts inversely related to the distance of the test locus from locus 0 . Anrep also reported that the gradients were bilaterially symmetrical; i.e., the amount of salivation elicited by stimulation of any locus on the contralateral side was almost identical to the amount elicited by stimulation of its ipsilateral homologue.

Decremental gradients along a somesthetic-spatial dimension in groups of men were subsequently reported for conditioned and pseudo-conditioned galvanic skin responses by Bass \& Hull (1934) and by Grant \& Dittmer $(1940 a, b)$, and for verbal reactions by Gibson (1939). All these American investigators used vibrotactile stimulation. There was a suggestion in Gibson's data that homologous loci on the trunk were to some extent similar in their response-evoking effects.

We have asked first, whether decremental gradients of generalization along a somesthetic-spatial dimension can be demonstrated in rats, and second, whether any degree of bilateral symmetry 'of gradients can be detected. Stimuli were electrical pulses, presented via subcutaneous electrodes; the response was a conditioned operant.

\section{Method}

Ss were three naive male albino rats, 4 months of age.

The method of stimulus presentation was modelled after that of Diamond et al (1964). On each side of the body, four evenly-spaced pairs of stainless steel electrodes were implanted beneath the skin, the most rostral pair just caudal to the shoulder, and the most caudal pair just rostral to the hip. Stimuli were generated by impressing biphasic $2 \mathrm{msec}$. pulses at $2 /$ sec. across the electrodes in a pair, from a Grass SD-5 stimulator. The cable from the SD-5 was interrupted by a slip-ring suspended over a hole in the top of the test chamber (Lehigh Valley Electronics 1417). A $38 \mathrm{~K}$ resistor in series with the animal served to diminish the effects of variations in tissue resistance. Peak current flow was $1 \mathrm{~mA}$; voltage drop varied around $1.3 \mathrm{~V}$. At this intensity, small muscle twitches were sometimes observed.

On the 10th-12th days after implantation, the rat was trained to press a lever for water while a particular locus (0) was stimulated. Starting with the 13th post-implantation day, 30-sec. S+ periods, during which
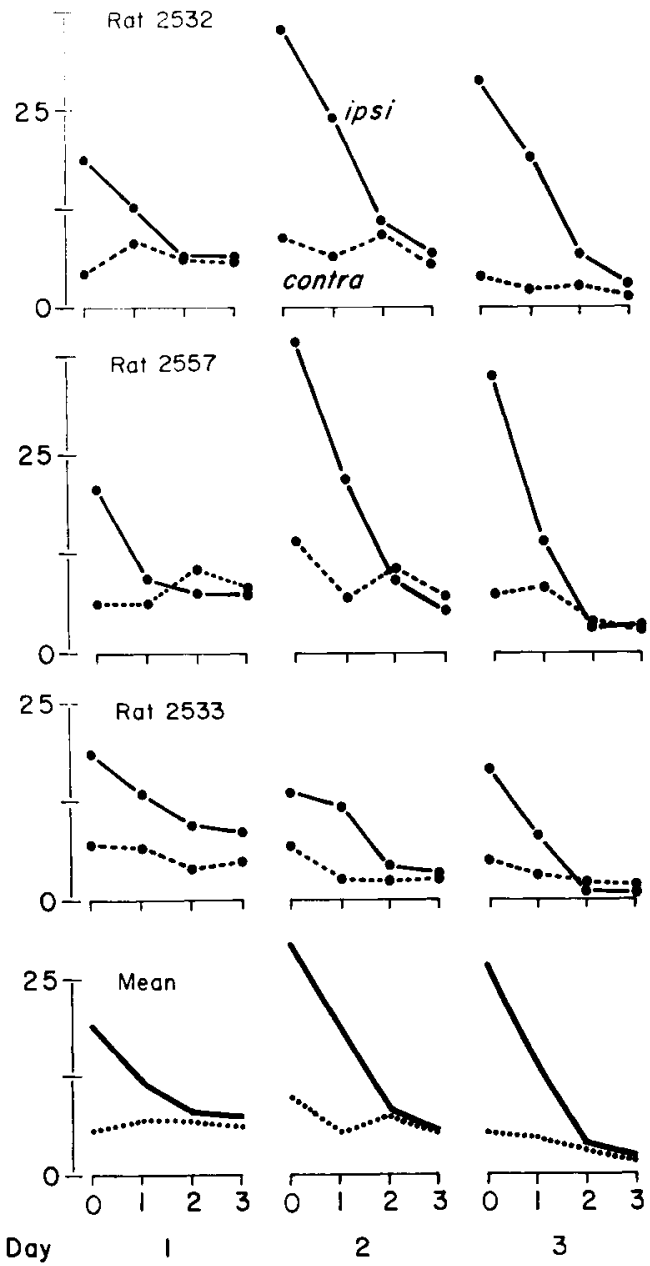

Fig. 1. Generalization gradients for each rat, by day. Solid lines: Responses $/ 30$ sec. as a function of distance of ipsilateral test locus from locus 0 . Dotted lines: Responses/30 sec. as a function of distance of contralateral locus from homologue of locus 0 . Bottom row: Group gradients, by day. 
stimulation was presented, alternated with 30-sec. time-out (TO) periods during which no stimulation occurred. Reinforcement was available on a VI-12 sec. schedule during $\mathrm{S}+$ periods, and never during TO. Locus 0 was the most caudal pair of electrodes on the right side for rat 2533, and the most rostral pair on the right side for rats 2532 and 2557 .

The rats were trained on consecutive days for 128 min. per day until the ratio of responses during S+ periods to responses during TO was $2: 1$. On the following three days, generalization-testing sessions were run, each lasting $128 \mathrm{~min}$. In each session a random half of the time-in periods were of the usual sort: locus 0 was stimulated, and reinforcement was available on the VI-12 schedule. In the remaining half of the time-in periods, reinforcement was not available and stimulation was presented to each of the eight loci eight times each. Order of test stimulations was varied to distribute sequence effects. Each locus was stimulated once in each cycle of eight successive test periods.

\section{Results and Discussion}

With minor exceptions (rat 2532, day 1; rat 2557, day 3), response rates to stimulation ipsilateral to locus 0 decreased monotonically with increasing distance from 0 , on each test day (Fig. 1). Ipsilateral locus was a significant source of variation for each rat on each day $(p<.001)$.

Response rates to contralateral stimulation were considerably lower than to ipsilateral stimulation, especially for the loci homologous to 0 and 1. There was a tendency toward monotonicity with continued testing, but contralateral locus was a significant source of variation only for rat 2557 on days $2(p<.05)$ and $3(p<.005)$. For the group, locus was not significant on day $1(F<1)$; significant on day $2(p<.025)$; and approached significance on day $3(.05<p<.10)$, when very few responses were emitted during contralateral stimulation. Collapsed over rats and days, the mean numbers of responses for the loci homologous to $0,1,2$, and 3 were $7.0,5.7,5.8$, and 4.5 , respectively $(.05<\mathrm{p}<.10)$.

Thus, clear-cut decremental gradients to ipsilateral stimulation have been obtained in the rat, similar to those obtained in dog and man with radically different stimulating, training, and testing techniques.

By contrast, the results on bilateral symmetry are ambiguous. The contralateral gradients were much lower in altitude than the ipsilateral ones. Although there was an approach toward monotonicity over sessions for all three rats, there was nothing like the coincidence of gradients on the two sides which Anrep found in individual dogs.

At present, we cannot say with certainty whether the obtained ipsilateral gradients have been influenced by peripheral factors. Depending upon the animal's position and the locus stimulated, the stimulus pulses sometimes evoked slight muscle twitches, with movement discernible at loci other than the stimulated one. Thus, bar-pressing might, to some extent, have become conditioned to the stimulation produced by such remote movement during acquisition periods. Work now in progress, with intensities which never evoke twitches, should help to clarify this point.

\section{References}

Anrep, G. V. The irradiation of conditioned reflexes. Proc. Roy. Soc. London, 1923, 94 B, 404-426.

Bass, M. J.. \& Hull, C. L. The irradiation of a tactile conditioned reflex in man. J. comp. Psychol., 1934, 17, 47-65.

Diamond, I. T., Randall, W., \& Springer, L. Tactual localization in cats deprived of cortical areas SI and SII and the dorsal columns. Psychon. Sci., 1964, 1, 261-262.

Gibson, Eleanor, J. Sensory generalization with voluntary reactions. J. exp. Psychol., 1939, 24, 237-253.

Grant, D. A., \& Dittmer, D. G. An experimental investigation of Pavlov's cortical irradiation hypothesis. J. exp. Psychol., 1940a, $26,299-310$.

Grant, D. A., \& Dittmer, D. G. A tactile generalization gradient for a pseudo-conditioned response. J. exp. Psychol., 1940b, 26, $404-412$.

Koupalov, P. La generalisation initiale et la specialisation consecutive des excitants conditionnels cutanes. Archives des Sciences biologiques (Petrograd), 1916, 19, 21-58.

\section{Notes}

1. From a paper read at Eastern Psychological Association meeting, April, 1966. Supported by a U. S. Public Health Service General Research Support grant, and by a grant from the United Health Foundation of Western New York (CG-65-UB-1).

2. Supported by P.H.S. research career program award (K3-MH5787) from the National Institute of Mental Health. 